\title{
Metabolic synthetic lethality
}

Molina et al.
have now
discovered an
inhibitor of
this process,
IACS-010759,
which is
specifically
effective in
cancer cells
that are
deficient in
glycolysis

Cancer cells adapt two key metabolic processes, glycolysis and oxidative phosphorylation (OXPHOS), to meet increased demands for energy to grow and survive. Whereas targeting elevated glycolysis has been researched extensively, targeting OXPHOS has been relatively unexplored, possibly because the extent to which tumours rely on OXPHOS for survival was unclear. As well as identifying multiple cancers that are highly dependent on OXPHOS, Molina et al. have now discovered an inhibitor of this process, IACS-010759, which is specifically effective in cancer cells that are deficient in glycolysis.

"Subpopulations of tumours depend on OXPHOS for biosynthetic and bioenergetics processes, opening the door for us to explore the biological mechanism(s) behind

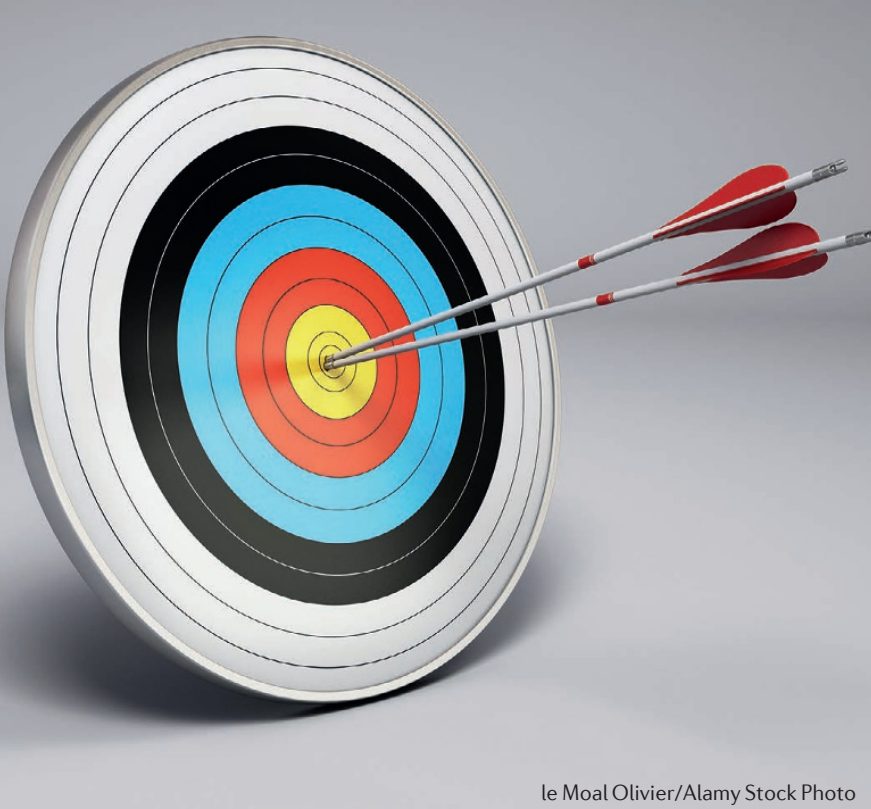

this process in cancer, and to exploit it therapeutically", explains Joseph Marszalek, senior author of the study.

The authors first screened known modulators of hypoxia-inducible factor 1- $\alpha$ (HIF-1 $\alpha$ ) then carried out a medicinal chemistry campaign to develop IACS-010759, an inhibitor of complex I of the mitochondrial electron transport chain. IACS-010759 inhibited the oxygen consumption rate and cell growth up to $100 \%$ in several cancer (pancreatic, lung, ovarian and breast) cell lines. Importantly, diploid cell lines were insensitive to IACS-010759 and showed no growth inhibition, confirming the selectivity of the compound.

Glycolysis and OXPHOS cooperate to maintain the cellular energetic balance, so genetic or pharmacological inhibition of OXPHOS can result in compensatory upregulation of glycolysis to maintain ATP levels. The authors hypothesized that tumour cells with a reduced capacity for glycolysis would thereby be more sensitive to OXPHOS inhibition. They tested IACS-010759 in a subpopulation of brain tumour cell lines (NB-1, Gli56 or D423 cells) with deficient glycolysis owing to lack of enolase 1 (ENO1) or phosphoglycerate dehydrogenase (PGD). Treatment resulted in $>70 \%$ reduction in viability and an increase in apoptosis in these cells, and this response was rescued by re-expression of ENO1 or PGD.

Exposure of acute myeloid leukaemia (AML) cells, which have a reported strong dependence on OXPHOS, to IACS-010759 for 3-7 days resulted in reduced viability, and this response was increased when glucose availability was restricted. The authors also tested the compound in primary AML blasts isolated from the peripheral blood of patients with relapsed or refractory AML ex vivo. IACS-010759 reduced viability and induced apoptosis in nearly all primary AML samples, but not in normal mononuclear cells, which suggested that IACS-010759 can selectively target leukaemic cells.

In vivo, treatment of mice carrying NB-1, Gli56 or D423-derived xenografts with IACS-010759 at doses of 5 or $10 \mathrm{mg} \mathrm{kg}^{-1}$ was well tolerated and resulted in tumour regression and extended overall survival. This antitumour activity was also confirmed in xenograft and syngeneic mouse models of AML.

Finally, Molina et al. characterized the molecular mechanism through which IACS-010759 exerts antitumour activity. Marszalek summarizes the key findings: "OXPHOS not only regulates energy production within cancer cells, but also aspartate production and utilization for nucleotide biosynthesis, which is similar to glycolysis. Depletion of these processes results in antitumour cell activities".

The development of IACS-010759 is the result of a drug discovery programme within the Center for Co-Clinical Trials (CCCT) and the Institute for Applied Cancer Science (IACS) at MD Anderson. IACS-010759 is in phase I clinical trials for both AML and solid tumours, and the first patient was enrolled in October 2016.

M. Teresa Villanueva

ORIGINAL ARTICLE Molina, J. R. et al. An inhibitor of oxidative phosphorylation exploits cancer vulnerability. Nat. Med. https://doi. org/10.1038/s41591-018-0052-4 (2018) 